\title{
Evacuation simulation of different flow ratios in low-density state
}

https://doi.org/10.1515/phys-2019-0009

Received October 30, 2018; accepted January 28, 2019

\begin{abstract}
The relationship between the factors of formation mechanism of stratification and the pedestrian ratio in low-density state has not been analyzed by the existing human flow evacuation simulation method, so that the simulation effect is poor. Thus, the evacuation simulation method for different flow ratios in low-density state is proposed to analyze the walking characteristics of the opposite pedestrians. On the basis of the random deviation grid gas model, the view field of pedestrian is introduced as one parameter. Considering the preference characteristics of pedestrians for the movement of open areas within the view field, the improved random deviation grid gas model is constructed. Through the model, the stratification characteristics of the opposite pedestrian flow in the simple channel scene are simulated. The results show that the proposed method can reproduce the characteristics of non-layering phenomenon of opposite pedestrian flow in low-density state. According to the probability of layer formation, the density of the opposite pedestrian flow is divided into five intervals. The opposite pedestrian flow in the critical density region is metastable, and is susceptible to interference. These results are consistent with the dynamic evolution of the actual opposite pedestrian flow.
\end{abstract}

Keywords: Low density, different flow ratios, evacuation, contrasting pedestrian flow, stratification, grid gas model

PACS: 02.70.-c; 07.05.Kf; 02.50.Tt

\footnotetext{
*Corresponding Author: Haitao Lian: School of Architecture,Tianjin University, Tianjin, 300072, China; School of Architectureand Art, Hebei University of Engineering, Handan, 056038, China, E-mail: lianhaitao@hebeu.edu.cn

Yike Hu: School of Architecture, Tianjin University, Tianjin, 300072, China, E-mail: huyike11@tju.edu.cn

R.D. Rohmat Saedudin: School of Industrial Engineering, Telkom University, 40257, Bandung, West Java, Indonesia,

E-mail: rdrohmat@telkomuniversity.ac.id
}

\section{Introduction}

The channel is an important part of the urban pedestrian transportation infrastructure and the most frequent pedestrian activity. It is widely distributed in indoor public places such as gymnasiums, cinemas, rail transit hubs and large supermarkets. The opposite pedestrian flow refers to the flow of people walking in the opposite direction of the walking facility, which is the most common type of pedestrian evacuation and the main cause of pedestrian treading accident [1]. The opposite pedestrian flow in the channel has been extensively studied as the simplest and most common phenomenon.

The most significant self-organizing phenomenon of the opposite pedestrian flow is the layer formation, also known as the line phenomenon [2]. Its formation is caused by the behavior of pedestrians following the same and avoiding opposite pedestrians. Most of the existing simulation models describe the difference selection of local paths by setting the movement rules, and reproduce the stratification phenomenon $[3,4]$. Existing research focuses on the re-emergence of stratification phenomena and the analysis of stratified quantities. However, the formation mechanism of stratification and its relationship with channel size, pedestrian density, pedestrian ratio of different trends, etc. have not been discussed in depth. It can be seen from the basic graph of the opposite pedestrian flow that the average velocity and flow of the opposite pedestrian flow are significantly increased after the formation of the stratification phenomenon. It can be seen that the in-depth understanding of the characteristics of stratification can provide theoretical basis for improving the comfort and safety of pedestrians [5].

The evacuation channel is used as the research scene, and the opposite pedestrian flow in the channel is selected as the research object. On the basis of the random deviation grid gas model, the view field of pedestrian is introduced as one parameter. Considering the preference characteristics of pedestrians for the movement of open areas within the view field, the improved random deviation grid gas model is constructed. Through the model, the stratification characteristics of the opposite pedestrian flow in 
the simple channel scene are simulated. This provides new idea for understanding the dynamic evolution process of pedestrian flow and the formation mechanism of stratification, and also has positive significance for improving the efficiency of pedestrian flow, optimizing channel design and ensuring pedestrian safety.

\section{Materials and methods}

\subsection{Model construction}

\subsubsection{Random deviation grid gas model}

In the basic random deviation grid gas model, the study scene is divided into $W \times L$ equal square grids [6], where $W$ is the width of the channel and $L$ is the length of the channel, as shown in Figure 1. The study scene included left-facing pedestrians and right-facing pedestrians, which are marked as hollow circles and solid circles, respectively. Pedestrians can move in any direction other than the rear according to certain probability [7]. $P_{1}$ is the probability that the pedestrian selects the grid on the left, $P_{2}$ is the probability of selecting the grid in the forward direction, and the $P_{3}$ is the probability of selecting the grid on the right. Each grid can only accommodate one pedestrian, and pedestrians cannot walk out of the boundaries of the scene. The left and right ends of the scene are set to periodic boundaries. After the pedestrian reaches the left (right) boundary, the scene is re-entered at the right (left) boundary. For analysis, set the channel length direction to the X-axis direction and the channel width direction to the $\mathrm{Y}$-axis direction, as shown in Figure 1.

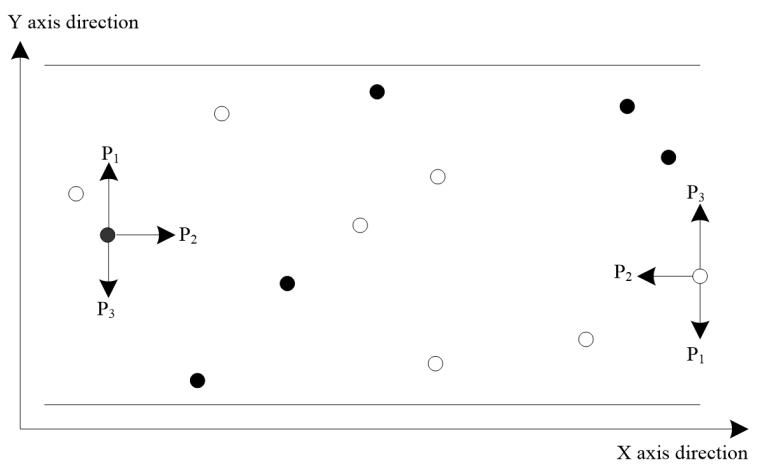

Figure 1: Simulation of pedestrian flow in a channel under periodic boundary conditions

When the simulation is updated, the next moving position of each pedestrian is determined by the occupancy of the three adjacent grids of the current grid. If an adjacent grid is not occupied, the pedestrian will move here with certain probability, which is called the transition probability [8]. $S_{1}, S_{2}$ and $S_{3}$ respectively indicate the occupation of the left, the front and the right side of the pedestrian, $S_{i}=0$ or $1(i=1,2,3)$. Where $S_{i}=0$ represents that the grid is not occupied, and $S_{i}=1$ is that there are people in the grid (or the grid is the boundary). According to the value combination, there are 8 kinds of movable states $\left(S_{1}, S_{2}\right.$ and $\left.S_{3}\right)$ of each pedestrian, and the basic transition probability of each state descending person selecting each adjacent lattice as the next target position is shown in Table 1.

Table 1: A basic transfer probability of pedestrians in each of the 8 neighborhood lattices

\begin{tabular}{llll}
\hline $\begin{array}{l}\text { State } \\
\text { number } \\
(\text { S1,S2,S3) }\end{array}$ & P1 (Left) & P2 (Ahead) & P3 (Right) \\
\hline $1(0,0,0)$ & $(1-\mathrm{D}) / 3$ & $\mathrm{D}+(1-\mathrm{D}) / 3$ & $(1-\mathrm{D}) / 3$ \\
$2(0,0,1)$ & $(1-\mathrm{D}) / 2$ & $\mathrm{D}+(1-\mathrm{D}) / 2$ & 0 \\
$3(0,1,0)$ & $1 / 2$ & 0 & $1 / 2$ \\
$4(0,1,1)$ & 1 & 0 & 0 \\
$5(1,0,0)$ & 0 & $\mathrm{D}+(1-\mathrm{D}) / 2$ & $(1-\mathrm{D}) / 2$ \\
$6(1,0,1)$ & 0 & 1 & 0 \\
$7(1,1,0)$ & 0 & 0 & 1 \\
$8(1,1,1)$ & 0 & 0 & 0 \\
\hline
\end{tabular}

As shown in Table 1, if the state of pedestrian is $(0,0$, 1), it means that there is no pedestrian on the left and front of the pedestrian, and the right side is adjacent to the grid (or boundary). The probability of selecting the left side of the grid is $P_{1}=(1-D) / 2$, the probability of selecting the adjacent grid in front is $P_{2}=D+(1-D) / 2$, the probability of selecting the right side of the grid is $P_{3}=0$. Where $D$ is the moving strength parameter, and $D \in[0.0,1.0]$. The greater its value is, the greater the walking speed of pedestrians is.

\subsubsection{Improved grid gas model based on open region mobility preferences}

In practice, when the pedestrian moves, he will comprehensively consider the distribution and number of twoway pedestrians in the view field, and then decide the direction and position of the next step. For example, pedestrians tend to choose more pedestrians and move toward fewer pedestrians, showing obvious follow-up behavior 
and avoidance behavior [9, 10]. At the same time, pedestrians are more inclined to move toward open areas with fewer pedestrians, thus avoiding mutual interference and traffic congestion between pedestrians as much as possible. Based on the grid gas model influenced by the view field, the selection preference characteristics of the open area [11] are analyzed, and the improved grid gas model is proposed to simulate the evolution process of the opposite pedestrian flow in the evacuation channel and stratification characteristics.

First, the pedestrian's field of view is divided into three square fields of view: the left front range, the front range, and the right front range [12]. The left front range and the right front range view area are each defined as the range of $m \times n$, and the front range view area is defined as the $m \times 1$ range. Where $m$ www is the length of the field of view and $n$ is the width of the field of view, as shown in Figure 2.

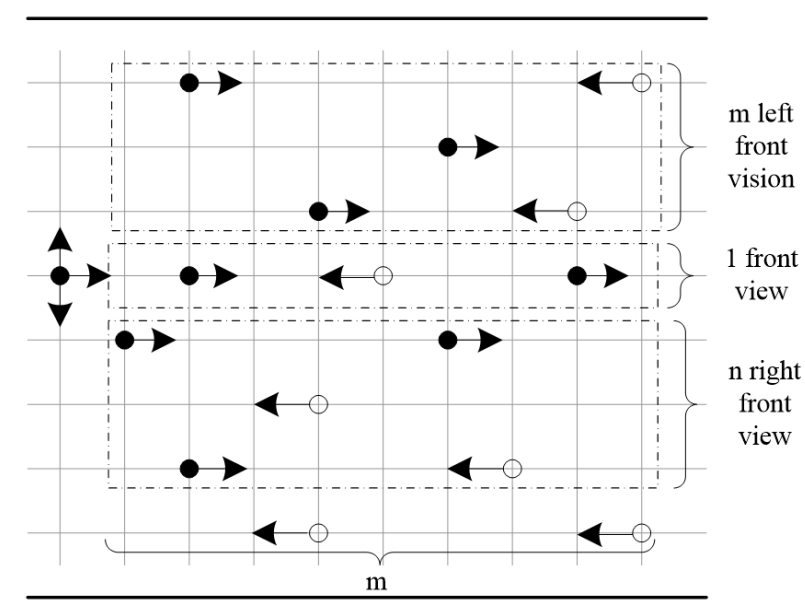

Figure 2: Sketch map of visual field for pedestrians in the passageway

The calculation method of the transition probability of the mobile preference of the open area is given [13], and the method is as follows:

Judging the movement state of the pedestrian according to whether the grid is on the left, front or right side of the grid where the pedestrian is currently located, and calculating the basic transition probability $P_{i}$ of moving to each adjacent grid according to Table 1 .

Calculate the number $T_{1}$ of people, the number $O_{1}$ of opposite rows and the number $E_{1}$ of spaces in the same direction of the left front view field; calculate the number $T_{2}$ of people, number $\mathrm{O}_{2}$ of opposite lines and number $E_{2}$ of spaces in the same direction of the front of the view field; calculate the number $T_{3}$ of people, the number $\mathrm{O}_{3}$ of op- posite lines and the number $E_{3}$ of spaces in the same direction of the right front view field.

Final transfer probability $\bar{P}$ :

$$
\bar{P}_{i}=P_{i}\left(\frac{E_{i}+T_{i}+1}{O_{i}+1}\right) / \sum_{i=1}^{3} P_{i}\left(\frac{E_{i}+T_{i}+1}{O_{i}+1}\right)
$$

Where, $\bar{P}_{i}$ represents the final transition probability of selecting each adjacent grid i, $i=1,2,3$, and $P_{i}$ represents the basic transition probability of the grid $i$;

Within each update step, the update order of the pedestrians is randomly determined. The final transition probabilities are sequentially calculated in order to determine and update the position of the pedestrian. When the pedestrian's location is updated, it is only necessary to calculate the final transition probability values of states 1,2 , 3 and 5. Compared with the Li model, the computational efficiency is significantly improved [14].

\subsection{Improved calculation method for channel evacuation}

The traditional empirical formula method does not calculate the detection and preparation time; it is assumed that the personnel start evacuation at the same time; the walking speed is estimated by uniform average value; the maximum capacity of the channel is given, and the personnel are continuously passed. These oversimplified assumptions do not match the actual ones, making the calculation error larger [15-21].

The improved empirical formula used in this paper breaks down the evacuation process into several processes: special case detection and personnel response, fixed positional exit, movement within the channel, and queuing or crowding at the exit of the channel. The time taken for each process is not calculated separately during the calculation, and the above processes are combined and calculated. The evacuation time formula is:

$$
t=t_{d}+t_{p}+t_{a}
$$

Where, $t$ is the total evacuation time; $t_{d}$ is the special case detection time;. $t_{p}$ is the personnel to detect the response; $t_{a}$ is the action time.

$$
t_{a}=t_{b}+t_{t}=t_{b}+\frac{P}{N R(W-2 L)}
$$

Where, $N$ is the number of channel ports; $R$ is the channel flow rate per unit time; $W$ is the channel length, and $L$ is the channel boundary width. 
Then the relationship between the flow rate $R$ and the flow density $D$ is

$$
R=k D(1-0.226 D)
$$

Where, $k$ is constant, and $D$ is the density of people.

\section{Experimental analysis}

\subsection{Evacuation channel scene settings}

Dividing an evacuation channel without internal obstacles into $W \times L$ square grids, the actual size of each grid is $0.4 \mathrm{~m} \times 0.4 \mathrm{~m}$ to ensure that each grid can hold up to one pedestrian. For example, when the evacuation channel has 50m length and $4 \mathrm{~m}$ width, $\mathrm{c}=125$ and $\mathrm{d}=10$, in order to meet the average walking speed of the actual pedestrian, the unit time step of the simulation corresponds to the physical time of $1 / 3 \mathrm{~s}$, that is, the pedestrian's moving speed is $1.2 \mathrm{~m} / \mathrm{s}$.

At the initial moment, the number of pedestrians in the scene is $N=E \times L \times \rho$, randomly distributed in the channel, where $\rho$ is the pedestrian density, $\rho \in[0.0,1.0]$. The number of pedestrians to the right side is $N_{\text {right }}=N \times f$, the number of pedestrians to the left side is $N_{\text {left }}=N \times$ $(1-f)$, and $f$ is the proportion of pedestrians to the right, $f \in[0.0,1.0]$. It is defined that the average speed of the opposite pedestrian flow is $v_{\text {average }}=N_{\text {forward }} / N$, and the flow rate $F_{\text {forward }}=v_{\text {average }} \times \rho$, as $N_{\text {forward }}$ is the number of pedestrians moving in the forward direction in the current simulation step.

\subsection{Basic diagram of the opposite pedestrian flow}

The simulation parameters are selected as follows $W=20$, $L=50, D=0.6, f=0.5, m=20, n=3$ and $\rho=0.3$. Each simulation runs 20,000 time steps, 5000 steps are selected for statistical data and the same parameters are repeated 50 times for averaging. The analysis results are shown in Figure 3.

It can be seen from Figure 3(a) that the average speed in the initial stage has rapidly increasing process as the density increases. Then, it slowly decreases as the density increases. When $\rho=0.4$, the speed will drop rapidly. This means that when pedestrian density exceeds the threshold, the opposite pedestrian flow will change from normal state to congested state; when the density is $\rho=0.48$, the average speed gradually decreases to zero as the den-

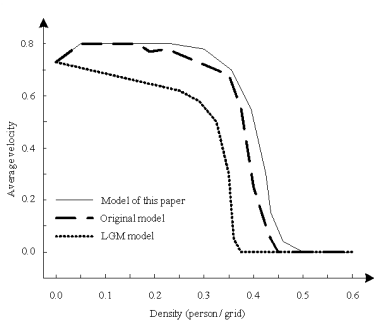

(a) Mean velocity density curve

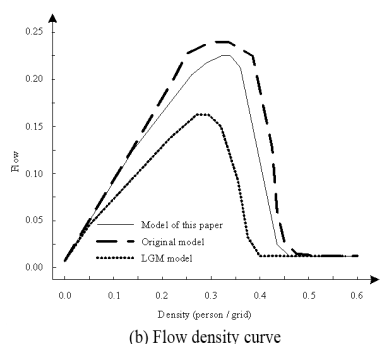

(b) Flow density curve
Figure 3: Pedestrian mean velocity and pedestrian flow and density curve

sity increases. At this time, the pedestrian flow is basically blocked.

At the same time, as can be seen from Figure 3(b), the flow rate also undergoes a process of increasing first, then suddenly decreasing, and finally gradually becoming zero as the density changes. This is consistent with the trend of the basic flow diagram of the opposite pedestrian flow obtained by other models. By comparing the basic model of the model and the original model, it is found that when the density is small $(\rho<0.2)$, the basic maps obtained by the two models have little difference; but when the density is $\rho \in[0.2,0.46]$,

the average velocity and flow rate of the model are slightly larger than the original model. This is because in this density interval, the pedestrians in this model have a stronger willingness to choose to open the open space than the original model. Each pedestrian has a greater speed of travel, making the average speed of the opposite pedestrian flow larger. When density is $\rho>0.46$, the opposite pedestrian flow in the model and the original model is in a deadlock state, and the average speed and flow rate are both zero.

\subsection{Evolution analysis of the opposite pedestrian flow}

The simulation parameters are selected as follows $W=20$, $L=50, D=0.6, f=0.5, m=20, n=3$ and $\rho=0.3$. Each simulation runs 20,000 time steps, 5000 steps are selected for statistical data and the same parameters are repeated 50 times for averaging. The evolution process of the opposite pedestrian flow in the channel under different simulation steps is shown in Figure 4.

As can be seen from Figure 4, the model of this paper can well reproduce the phenomenon of pedestrian flow stratification. As shown in Figure 4(a), at the initial time simulation time step, pedestrians are randomly distributed in the channel; at that time, six layers are ba- 


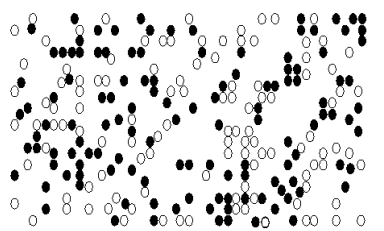

(a) $\mathbf{t}=\mathbf{0}$

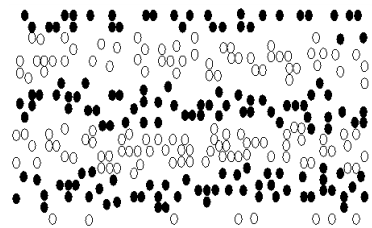

(c) $\mathbf{t}=798$

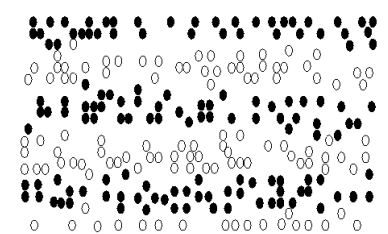

(b) $\mathrm{t}=228$

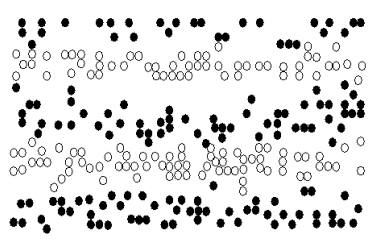

(d) $\mathrm{t}=998$
Figure 4: Evolution process of pedestrian flow in different channels under different simulation steps

sically formed in the channel, as shown in Figure 4(b); with the advancement of the simulation step The layer on the lowermost side to the left gradually dissipates, and the pedestrians in this layer gradually move to the nearest same layer, as shown in Figure 4(c); finally, when $t=998$, five stable layers are formed in the channel, shown in Figure $4(\mathrm{~d})$. The change in the number of stratifications is consistent with the conclusion that the number of stratified pedestrian flow stratifications indicated by Helbing et al. is dynamic changes.

\subsection{Critical density analysis of opposite pedestrian flow}

Through simulation analysis, it is found that there is a density interval that will definitely form stratification phenomenon or cause blockage, which is defined as critical density interval. In the interval, the opposite pedestrian flow is in a metastable state, which is easily disturbed by the internal and external factors of the system and undergoes phase change. When the density is greater than the upper limit of the critical density interval, the phase change will definitely occur.

To ensure safe and efficient evacuation of pedestrians, traffic is artificially controlled to ensure that the system does not reach the critical state of congestion. However, the precondition is to grasp the specific data of the critical density interval of the opposite pedestrian flow. To this end, through a large number of simulation experiments, the number of occurrences of each state at different pedestrian densities are counted to analyze the range of critical density intervals. According to the previous literature analysis, at low density, the pedestrian flow evolves into free-flow state or layering form; at high density, the pedestrian flow evolves into layering form or congested state. Therefore, only the number of occurrences of the layering form will be counted.

First, according to the characteristics of the layering form, it is defined that the proportion of the same-way pedestrians in the $\mathrm{X}$-axis direction of the channel exceeds $90 \%$, and the ratio of the number to the total number $\mathrm{W}$ of pedestrians exceeds $90 \%$, so the layered form can appear; when the average speed of pedestrians in the channel $v_{\text {average }}=0$, the channel is in a congested state.

The system simulation parameters is taken as $\rho=$ $\{0.01,0.02,0.03, \cdots, 0.6\}, W=20, L=50, D=0.6$, $f=0.5, m=20, n=3$. Each simulation is at least 20,000 steps to ensure that the system reaches the steady state, and after 20,000 steps, it is judged whether layered form is formed. The simulation of the same parameters is repeated 500 times, and the number of stratification phenomena is counted, and the probability $P_{\text {lane }}$ of formation of stratification phenomena at different densities is calculated, as shown in Figure 5.

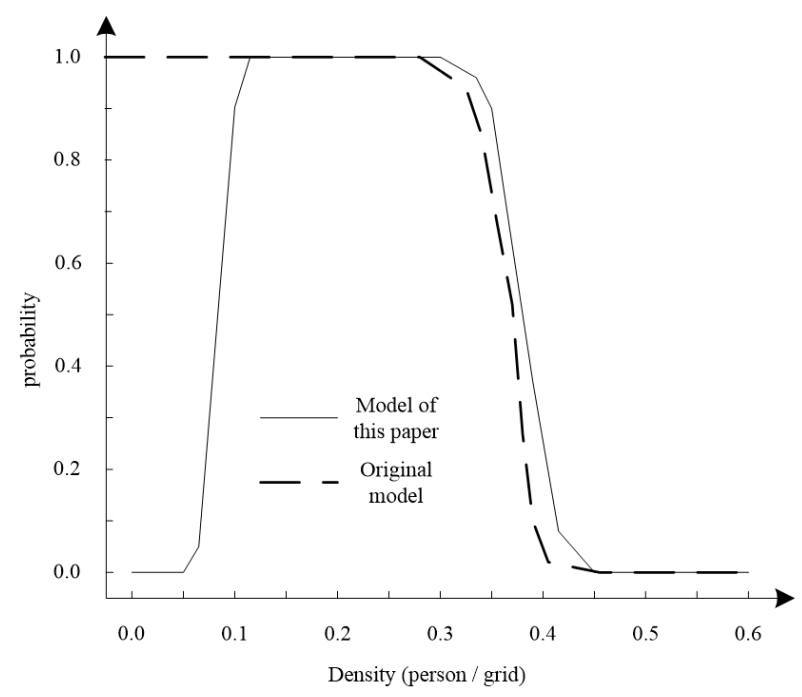

Figure 5: Probability maps of stratified phenomena with different density of pedestrian flow

It can be seen from Figure 5 that the density value $\rho$ can be divided into five intervals according to the probability $P_{\text {lane }}$ distribution formed by the stratification phenomenon of the opposite pedestrian flow, as follows:

When $0<\rho<0.05$, it is low density interval. The opposite pedestrian flow is always in a free-flow state, and there is no stratification, that is $P_{\text {lane }}=0.0$. By observing the simulation evolution map, it is found that within this density interval, the local extent of the channel will 
form an unstable layered shape, but as the simulation time advances, the layered shape will also disappear. Even if stratification occurs locally, the stratification pattern is destroyed because the pedestrian is attracted by the open area.

When $0.05<\rho \leq 0.08$, it is called the low-density critical interval, and the opposite pedestrian flow is in metastable state, that is, the opposite pedestrian flow will form stable layered shape with a certain probability. If the layered form is not formed, the pedestrian flow will be in free flow state, and the pedestrians walk freely within the passage. When $\rho=0.06, P_{\text {lane }}=0.068$; when $\rho=0.07$, $P_{\text {lane }}=0.892$. This is because when $0.05<\rho \leq 0.08$, the number of pedestrians in the view field gradually increases, and the effects of co-directional attraction and opposite rejection gradually become apparent. The local layered morphology is easily expanded into layered morphology of the entire channel. The greater the pedestrian density is, the more likely the local layered morphology is in the channel.

When $0.08<\rho \leq 0.32$, it is called the medium density interval; as $0.32<\rho \leq 0.46$, it is called the high density critical interval; as $\rho>0.46$, it is called the high density interval; the above three intervals are not in the research scope of this paper and will not be described in detail.

\subsection{Influence analysis of formation probability of layering phenomenon of opposite pedestrian flow}

According to the analysis of the previous section, this section discusses the relationship between the formation of the layering phenomenon and the model parameters such as the channel width $\mathrm{W}$, the length $\mathrm{L}$, the moving intensity $\mathrm{D}$, the right pedestrian flow ratio $f$, and the view field range $m, n$.

\subsubsection{The influence of system geometry on layering}

In this section it is mainly analyzed the influence of channel width $W$ and length $L$ on the formation of human flow layering.

The relationship between the formation probability $P_{\text {lane }}$ of layering phenomenon and the density $\rho$ of pedestrians under different widths of the channel is shown in Figure 6. When $W$ is small, the value of the low density critical interval is small. At the same density, the smaller $W$ is, the larger the $P_{\text {lane }}$ value is. This is because the narrow passage will make the coverage of the view field larger, and its effect on the same direction and opposite direction will be more obvious. At the same density, as the $W$ value increases, the $P_{\text {lane }}$ value decreases slightly because the number of spaces available for pedestrian position swapping is reduced.

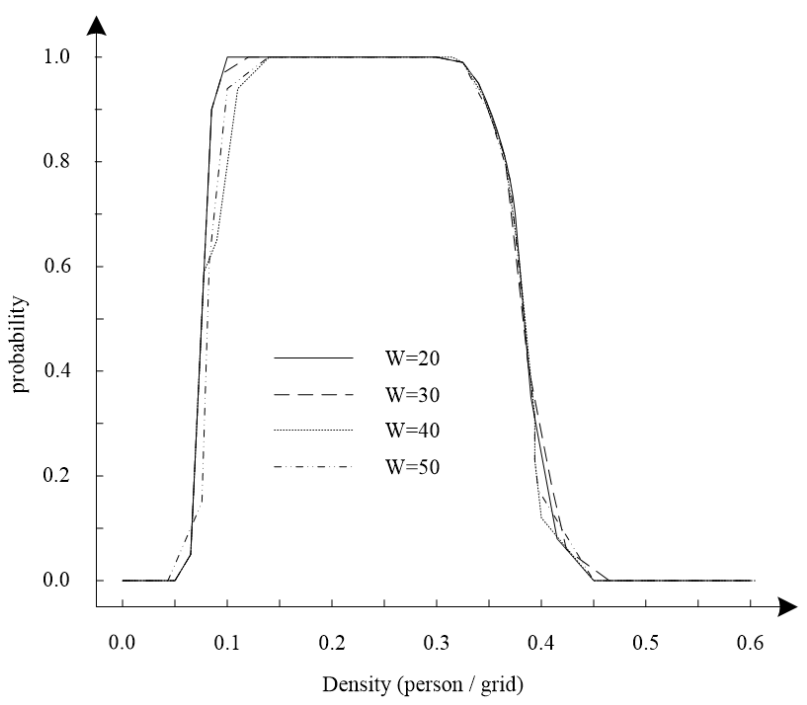

Figure 6: Probability of pedestrian density and stratification formation at different widths

Under the condition of different channel lengths, the relationship between the probability $P_{\text {lane }}$ formed by the stratification phenomenon and the pedestrian density $\rho$ is shown in Figure 7. It can be seen that in the low-density critical interval, the change of $L$ has little effect on $P_{\text {lane }}$.

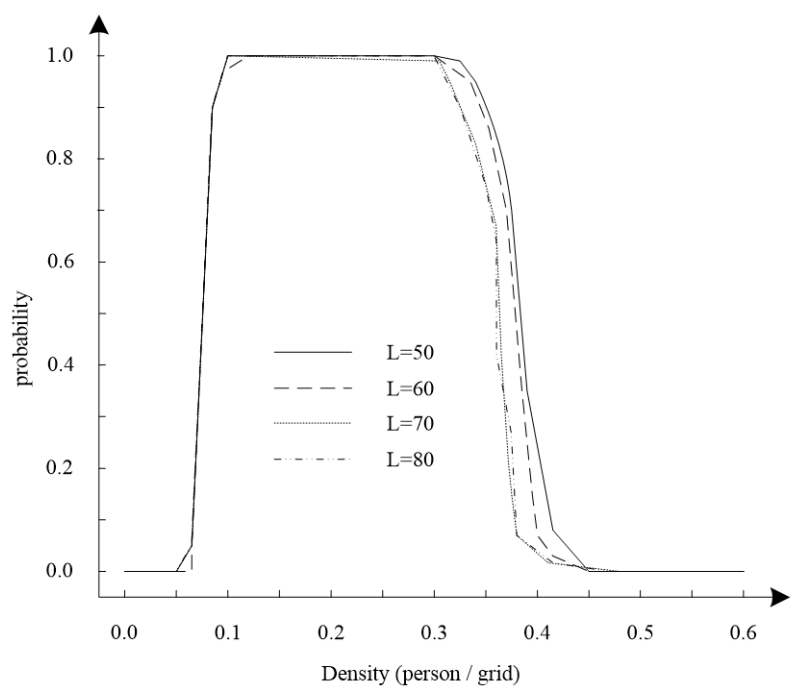

Figure 7: Probability density diagram of pedestrian density and seal formation at different lengths 


\subsubsection{Impact of $\boldsymbol{D}$ and $\boldsymbol{f}$ on stratification}

In this section it is mainly analyzed the influence of the moving intensity parameter $D$ and the proportional parameter $f$ of the right pedestrian flow on the stratification phenomenon of human flow. Figure 8 is the graph of the probability $P_{\text {lane }}$ of pedestrian density $\rho$ and stratification formation at $D=0.0,0.2,0.4,0.6,0.8$. It can be seen from Figure 8 that the change $D$ has a certain influence on the range of two critical density intervals. In the lowdensity critical interval, as the value increases, the value $P_{\text {lane }}$ at the same density will decrease slightly. This is because the larger the value, the smaller the willingness of the lateral offset, and the smaller the adjustment probability of the position in the Y-axis direction between the pedestrians, which allows the pedestrian flow at low density to maintain a free flow state with difficult formation. Muramatsu will explain $D$ as the willingness of pedestrians to move forward. The greater the value, the more pedestrians tend to walk forward, and the faster they will travel. Therefore, the analysis of the relative pedestrian flow from the perspective of the formation probability of the stratification phenomenon can also lead to the phenomenon of "faster is slower" proposed by Helbing et al.

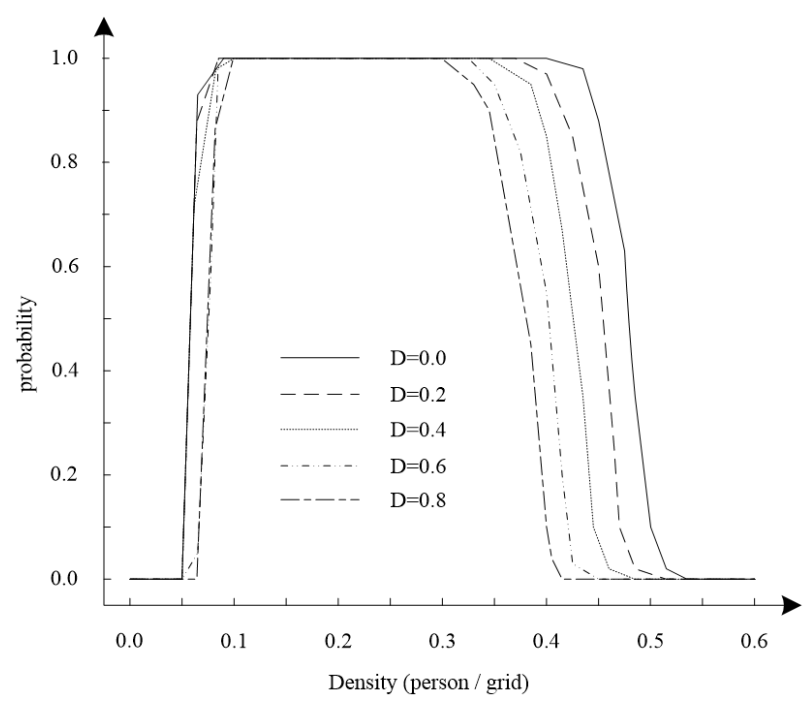

Figure 8: Pedestrian density and stratified formation probability map with different $D$ values

It can be seen from Figure 9 that in the low density critical interval, when the density is the same, the increase of the f value causes the $P_{\text {lane }}$ value to decrease slightly. This is mainly because the unbalanced opposite pedestrian flow is more likely to form layered form in this case.

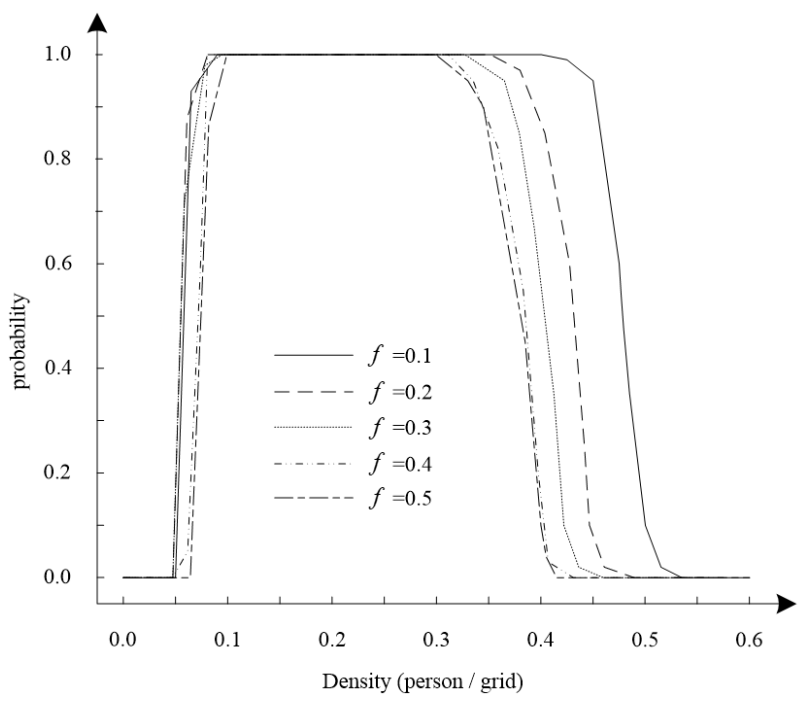

Figure 9: Pedestrian density and stratified formation probability map with different $f$ values

\subsubsection{Impact of range parameters on stratification}

The effect of the parameters $m$ and $n$ of view field on the stratification phenomenon is discussed in this section.

When the length of visual field $m=5,10,15,20$, the curve of the pedestrian density $\rho$ and the formation probability $P_{\text {lane }}$ of the layering phenomenon is as shown in Fig. 10. It can be seen from Figure 10 that the change has a certain influence on the low density critical interval. When the $m$ value increases, both density critical intervals are shifted to the negative direction of the $\mathrm{X}$ axis, and the offset of the low density critical interval is more significant. In the low-density critical interval, the $m$ value is larger and the $P_{\text {lane }}$ value is larger in the case of the same density.

When the width of view field is $n=1,2,3,4,5,6$, the curve of pedestrian density $\rho$ and probability $P_{\text {lane }}$ is shown in Figure 11. As can be seen from Figure 11, the $n$ change has significant impact on the low density critical interval. When the $n$ value increases, the low density critical interval is shifted in the negative direction of the $\mathrm{X}$ axis. The smaller the $n$ value is, the larger the offset is. In the low-density critical interval, under the same density $\rho$, the larger the $n$ value is, the larger the $P_{\text {lane }}$ value is. This makes the field of view limited at low density and is less prone to delamination. This phenomenon is especially noticeable when $n=1$; when $n=0$, the model degenerates into basic grid gas model, and no delamination occurs at any density. Comparing Figures 6 and 7, it can be seen that the visual field range parameter has greater influence on the formation of the layered shape of the opposite pedestrian flow than the system geometric size. 


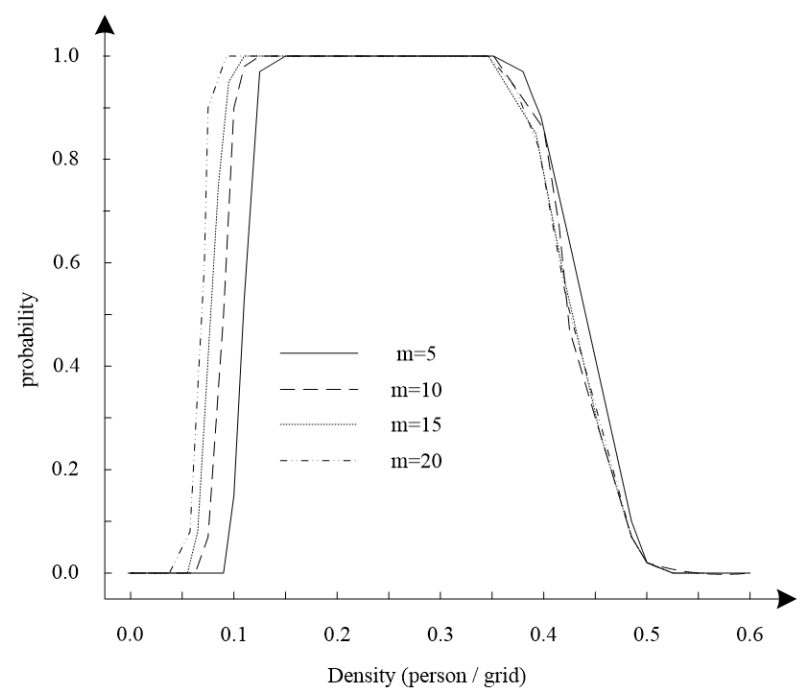

Figure 10: Pedestrian density and stratified formation probability map with different $m$ values
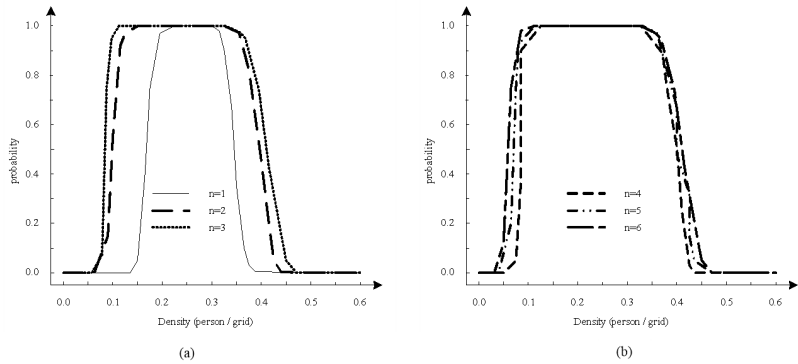

Figure 11: Pedestrian density and stratified formation probability map with different $n$ values

\section{Conclusions}

Based on the observation of the walking characteristics of the opposite pedestrians in the evacuation channel, based on the grid gas model of the pedestrian's visual field, the characteristics of the mobile preference in the open area are introduced, and the improved grid gas model is proposed. The update rules of the model fully reflect the characteristics of pedestrians' following direction, opposite avoidance, and open regional mobility preferences.

Based on the improved grid gas model proposed in this paper, a large number of simulation experiments are carried out on the opposite pedestrian flow in the channel. The evolution process of the opposite pedestrian flow in low density state is emphasized to study the formation probability of the layering phenomenon of the opposite pedestrian flow in the low density state and its relationship with the channel geometry, the moving intensity pa- rameter, the right pedestrian flow ratio and the view field. Main conclusions are as follows

Under different density states, the opposite pedestrian flow of this model presents three evolution processes: free flow, layered form and blockage. The model of this paper can reproduce the characteristics of non-layering phenomenon of opposite pedestrian flow in low density state, which is more realistic than the existing model.

According to the formation probability of stratification phenomenon of opposite pedestrian flow, the flow density is divided into five sections: low, medium, high, low critical and high critical. The evolution process of pedestrian flow in different intervals is different. The opposite pedestrian flow in the critical density region is in metastable state, which is susceptible to interference and phase changes.

The influence of channel geometry on the formation of stratification phenomenon in low density state is not significant.

The width of the view field has great influence on the formation of the stratification phenomenon of the opposite pedestrian flow in the low density state.

\section{References}

[1] Yao C.Z., Liu X.F., Lin J.N., Study on pedestrian flow evacuation with individual-guidance mechanism, Kybernetes, 2016, 45, 772-787.

[2] Carrillo J.A., Martin S., Wolfram M.T., An improved version of the hughes model for pedestrian flow, Math. Mod. Meth. Appl. Sci., 2016, 26, 671-697.

[3] Li M.H., Yuan Z.Z., Xu Y., Randomness analysis of lane formation in pedestrian counter flow based on improved lattice gas model, Acta Phys. Sin., 2015, 64, 18903-018903.

[4] Kubera P., Felcman J., On a numerical flux for the pedestrian flow equations, J. China Acad. Electr. Inform. Technol., 2015, 11, 7996.

[5] Hu J., Li Z.W., Zhang H., Wei J., You L., Chen P., Experiment and simulation of the bidirectional pedestrian flow model with overtaking and herding behavior, Int. J. Mod. Phys. C, 2015, 26, 0131.

[6] Lei Q., Based on the multisim voter circuit design and analysis of three people, Automat. Instrument., 2016, 2, 19-29.

[7] Zhou M., Dong H., Wang F.Y. Wang Q.L., Yang X.X., Modeling and simulation of pedestrian dynamical behavior based on a fuzzy logic approach, Informat. Sci., 2016, 360,112-130.

[8] Agnelli J.P., Colasuonno F., Knopoff D. A kinetic theory approach to the dynamics of crowd evacuation from bounded domains, Math. Mod. Meth. Appl. Sci., 2015, 25,109-129.

[9] Albi G., Bongini M., Cristiani E., Kalise D.., Invisible control of self-organizing agents leaving unknown environments, Siam J. Appl. Math., 2016, 76, 1683-1710.

[10] Goodwin M., Granmo O.C., Radianti J., Escape Planning in Real- 
istic Fire Scenarios with Ant Colony Optimisation, Appl. Intel., 2015, 42, 24-35.

[11] Dridi M.H., Simulation of high density pedestrian flow: microscopic model, J. Pow. Sup., 2015, 03, 81-95.

[12] Cao S., Song W., Lv W., Modeling pedestrian evacuation with guiders based on a multi-grid model, Phys. Lett. A, 2016, 380, 540-547.

[13] Fu L., Song W., Lo S., A fuzzy-theory-based behavioral model for studying pedestrian evacuation from a single-exit room, Phys, Lett. A, 2016, 380, 2619-2627.

[14] Xue S., Jia B., Jiang R. Shan J., Pedestrian evacuation in view and hearing limited condition: the impact of communication and memory, Phys Lett. A, 2016, 380, 3029-3035.

[15] Luo L., Fu Z., Zhou X., Zhu K., Yang H., Yang L., Fatigue effect on phase transition of pedestrian movement: experiment and simulation study, J. Stat. Mech. Theory and Exp., 2016, 2016, 103-401.

[16] Kylili A., Fokaides P.A., loannides A., Kalogirou S., Environmental assessment of solar thermal systems for the industrial sector, J. Clean. Prod., 2018, 176, 99-109.
[17] Bortolan M.C., Rivero F., Non-autonomous perturbations of a non-classical non-autonomous parabolic equation with subcritical nonlinearity, Appl. Math. Nonlin. Sci., 2017, 2(1), 31-60.

[18] Gao W., Baig A.Q., Ali H., Sajjad W., Farahani M.R., Margin based ontology sparse vector learning algorithm and applied in biology science, Saudi J. Biol. Sci., 2017, 24(1), 132-138.

[19] Liu Z., Peng W., Zare Y., Hui D., Rhee K.Y., Predicting the electrical conductivity in polymer carbon nanotube nanocomposites based on the volume fractions and resistances of the nanoparticle, interphase, and tunneling regions in conductive networks, Rsc Advances, 2018, 8(34), 19001-19010.

[20] Lakshminarayana G., Vajravelu K., Sucharitha G., Sreenadh S., Peristaltic slip flow of a bingham fluid in an inclined porous conduit with joule heating, Appl. Math. Nonlin. Sci., 2018, 3(1), 4154.

[21] Khan A.M., Yusoff I., Abu Bakar N.K., Abu Bakar A.F., Alias Y., Mispan M.S., Accumulation, uptake and bioavailability of rare earth elements (rees) in soil grown plants from ex-mining area in Perak, Malaysia, Appl. Ecol. Envir. Res., 2017, 15(3), 117-133. 